\title{
Primary Hepatic Adenosquamous Carcinoma Associated with Primary Sclerosing Cholangitis
}

\author{
Kentaro Yamao ${ }^{a}$ Mamoru Takenaka ${ }^{a}$ Hajime Imai ${ }^{a}$ Atsushi Nakai ${ }^{a}$ \\ Shunske Omoto ${ }^{a}$ Ken Kamata ${ }^{a}$ Kosuke Minaga ${ }^{a}$ Takeshi Miyata ${ }^{a}$ \\ Toshiharu Sakurai $^{\mathrm{a}}$ Tomohiro Watanabe $^{\mathrm{a}}$ Naoshi Nishida $^{\mathrm{a}} \quad$ Ippei Matsumoto $^{\mathrm{b}}$ \\ Yosihumi Takeyama ${ }^{b}$ Takaaki Chikugo $^{c}$ Masatoshi Kudo $^{a}$

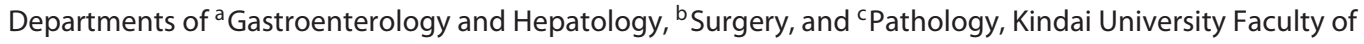 \\ Medicine, Osaka-Sayama, Japan
}

\section{Keywords}

Adenosquamous carcinoma · Primary sclerosing cholangitis · Liver tumor · Cholangiocarcinoma · Liver abscess $\cdot$ Ulcerative colitis

\begin{abstract}
Introduction: Primary sclerosing cholangitis (PSC) is a chronic cholestatic liver disorder characterized by multiple fibrotic strictures of the bile duct. More than $40 \%$ of deaths in PSC patients are related to malignant tumors, including cholangiocarcinoma. Primary hepatic adenosquamous carcinoma (ASC) is a rare subtype of cholangiocarcinoma containing adenocarcinoma $(A C)$ and squamous cell carcinoma (SCC) components, with a poorer prognosis than other cholangiocarcinomas. We report the first case of a hepatic ASC in a patient with PSC. Case Report: A 28-year-old man was referred for diagnosis and treatment of a liver abscess suspected by contrast-enhanced computed tomography (CECT). He had a history of ulcerative colitis and PSC. Abdominal CE-CT revealed a $60-\mathrm{mm}$-diameter ring-shaped mass with central necrosis in the left lobe. Magnetic resonance imaging demonstrated a poorly circumscribed low-signal-
\end{abstract}

\section{KARGER}

(C) 2017 S. Karger AG, Basel

E-Mail karger@karger.com

www.karger.com/ocl intensity mass in T1-weighted imaging and a high-signalintensity mass with a scattered low-signal-intensity area in T2-weighted imaging. Abdominal ultrasonography showed a hypoechoic component with a diffuse hyperechoic area in the tumor. Ultrasound-guided biopsy and histological examination showed tumor cells with both squamous and glandular differentiation. Left lobectomy was performed. Microscopic examination revealed 2 components, including moderately differentiated AC and well-differentiated SCC. The final diagnosis was hepatic ASC. Conclusion: This is the first reported case of hepatic ASC in a patient with PSC. Patients with PSC should be recognized as being at a risk of not only general cholangiocarcinoma, hepatocellular carcinoma, and metastatic liver tumor, but also ASC.

(c) 2017 S. Karger AG, Basel

\section{Introduction}

Primary sclerosing cholangitis (PSC) is a chronic cholestatic liver disorder characterized by multiple fibrotic strictures of the intra- and extrahepatic biliary tree. Previous studies have shown that $>40 \%$ of deaths in patients 
Fig. 1. a Contrast-enhanced abdominal computed tomography. A mass $62 \times 45 \mathrm{~cm}$ in diameter showing a low-density tumor with an irregularly enhanced rim in the left lobe. b Magnetic resonance imaging. T1weighted image demonstrated a poorly circumscribed low-signal-intensity mass. c T2-weighted image demonstrated a highsignal-intensity mass with a scattered lowsignal-intensity area in the left lobe. d Ultrasonography showed a hypoechoic component with a diffuse hyperechoic area in the tumor. e Contrast-enhanced ultrasonography showed an avascular area in the tumor.
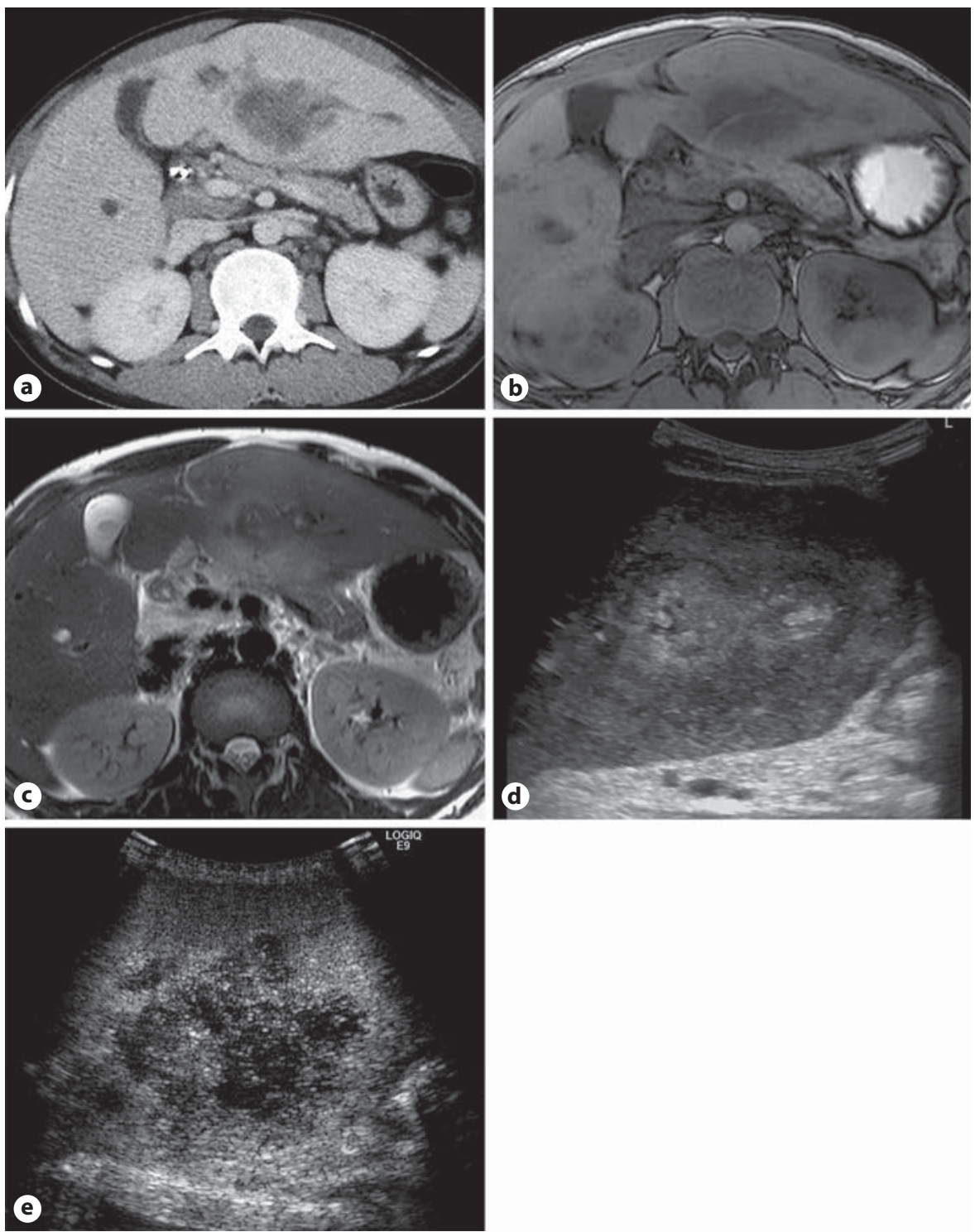

with PSC are related to cholangiocarcinoma, gallbladder carcinoma, or colorectal carcinoma $[1,2]$. Cholangiocarcinoma is the most relevant of these, with a lifetime risk in PSC patients of around 7-14\% and a prognosis including a 5 -year survival of $<10 \%$ [2].

Primary hepatic adenosquamous carcinoma (ASC) is defined as a rare subtype of cholangiocarcinoma, accounting for $2-3 \%$ of cholangiocarcinomas [3-5]. ASC contains both adenocarcinoma (AC) and squamous cell carcinoma (SCC) components, tends to present more aggressive clinicopathologic features, and has a poorer prognosis than other cholangiocarcinomas $[6,7]$. Barr and Hancock [8] first reported a case of primary hepatic

Hepatic Adenosquamous Carcinoma with PSC
ASC in 1975, since when several cases of histologically defined primary hepatic ASC have been reported in the literature [3, 9-12]; however, none have been reported in patients with PSC. We herein report the first case of hepatic ASC in a patient with PSC.

\section{Case Report}

A 28-year-old man was referred for diagnosis and treatment of a liver abscess suspected by abdominal contrast-enhanced computed tomography (CT). The patient's chief complaints were fever and epigastric pain. He was a nonsmoker and had no history of alcohol abuse. He had a 10-year history of ulcerative colitis and a 
Fig. 2. a AC; HE stain. b Immunostaining for carcinoembryonic antigen revealed cytoplasm-positive staining for AC. c SCC; HE stain. d Immunostaining for cytokeratin 5/6 revealed cytoplasm-positive staining for SCC. AC, adenocarcinoma; HE, hematoxylin and eosin; SCC, squamous cell carcinoma.
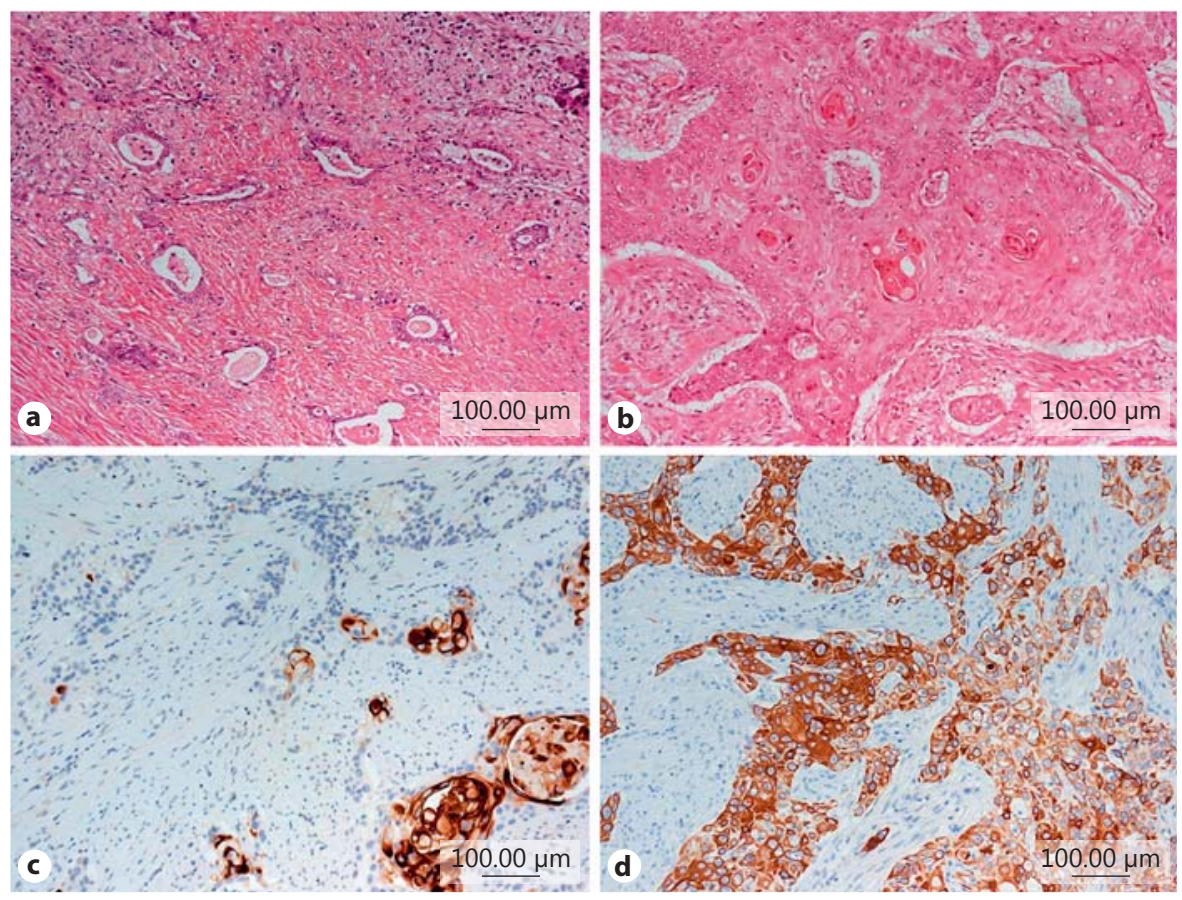

3-year history of PSC. Endoscopic biliary drainage was performed regularly to exchange the biliary stent in the left bile duct with the stenosis due to PSC.

The results of relevant laboratory studies were total bilirubin 3.4 $\mathrm{mg} / \mathrm{dL}$ (normal $0.3-1.2 \mathrm{mg} / \mathrm{dL}$ ), aspartate transaminase $72 \mathrm{IU} / \mathrm{L}$ (normal 13-33 IU/L), alanine transaminase 56 IU/L (normal 8-42 $\mathrm{IU} / \mathrm{L}$ ), alkaline phosphatase 1,554 U/L (normal 106-322 U/L), albumin $2.9 \mathrm{~g} / \mathrm{dL}$ (normal $4.1-5.1 \mathrm{~g} / \mathrm{dL}$ ), C-reactive protein $8.07 \mathrm{mg} / \mathrm{dL}$ (normal $0-0.14 \mathrm{mg} / \mathrm{dL}$ ), white blood cells $16,800 / \mathrm{mL}$ (normal $2,800-9,200 / \mathrm{mL}$ ), prothrombin time international normalized ratio 1.32, indocyanine green retention rate at $15 \mathrm{~min} 26.0 \%$ (normal $0-10 \%$ ), carcinoembryonic antigen $6.1 \mathrm{ng} / \mathrm{mL}$ (normal $<5.0 \mathrm{ng} / \mathrm{mL}$ ), carbohydrate antigen 19-9,290 U/mL (normal $<37 \mathrm{U} / \mathrm{mL}$ ), and SCC antigen $5.1 \mathrm{ng} / \mathrm{mL}(0-1.5 \mathrm{ng} / \mathrm{mL})$. The patient tested negative for hepatitis $\mathrm{B}$ viral surface antigen and hepatitis $\mathrm{C}$ viral antibody.

Abdominal contrast-enhanced CT showed an enhanced ringshaped mass measuring $62 \times 45 \mathrm{~mm}$ in diameter with central necrosis in the left lobe (Fig. 1a). Magnetic resonance imaging demonstrated a poorly circumscribed low-signal-intensity mass in T1weighted images (Fig. 1b) and a high-signal-intensity mass with a scattered low-signal intensity area in T2-weighted images (Fig. 1c). Positron emission tomography showed increased ${ }^{18} \mathrm{~F}$-fluorodeoxyglucose uptake (maximum standardized uptake value: 9.59) in the tumor. These findings, especially the inflammatory reaction, led to suspicion of a liver abscess, but increasing tumor markers indicated a malignant liver tumor.

Abdominal ultrasonography showed a hypoechoic mass with a partial hyperechoic area (Fig. 1d), and contrast-enhanced ultrasonography showed a heterogeneous hypoechoic tumor with a partially avascular area (Fig. 1e). Ultrasonography-guided biopsy was performed, and histological examination revealed tumor cells with both squamous and glandular differentiation. The diagnosis was ASC.
Left lobectomy was performed. The cut surface of the resection specimen showed a yellowish-white solid tumor, measuring $65 \times$ $55 \times 45 \mathrm{~mm}$, with partial necrosis. Microscopic examination revealed 2 components, including moderately differentiated AC (Fig. 2a) and well-differentiated SCC (Fig. 2b). The SCC component was predominant over the AC component and occupied $>80 \%$ of the tumor. There was also a transitional area between the 2 components. Immunohistochemistry revealed carcinoembryonic antigen and cytokeratin 7 expression in most glandular and transitional epithelia (Fig. 2c), but no expression in the stratified squamous epithelia, whereas cytokeratin 5/6 was expressed in most of the SCC (Fig. 2d). A final diagnosis of hepatic ASC was made on the basis of the histological and immunohistochemical findings. Periductal concentric fibrosis of the bile ducts (onion skin-like appearance) suggested the presence of PSC (Fig. 3).

Recurrence was detected in the liver by follow-up examination 1 month postoperatively, and the patient died of ASC recurrence 2 months after surgery.

\section{Discussion}

PSC is a chronic inflammatory liver disease of unknown etiology, primarily targeting cholangiocytes at any portion of the biliary tree. Liver transplantation is currently the only effective treatment. PSC shows a close association with inflammatory bowel disease, mainly ulcerative colitis. As in many chronic inflammatory conditions, PSC may be complicated by cancer development, which accounts for $>40 \%$ of deaths in patients with PSC 


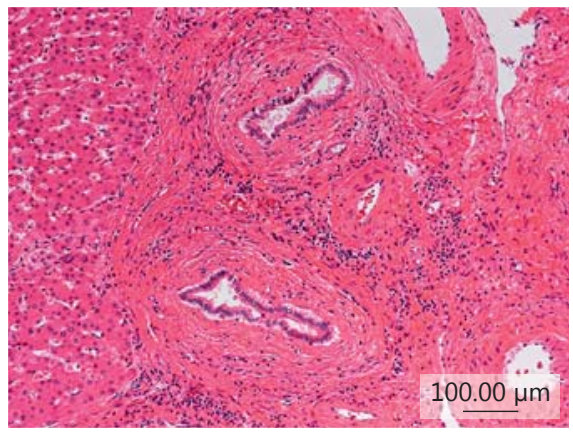

Fig. 3. Periductal concentric fibrosis of the bile ducts was present (onion skin-like appearance).

$[1,2,13]$. Cholangiocarcinoma, gallbladder carcinoma, and colorectal carcinoma have been variably associated with PSC, with prevalences of up to 13-14\% [14].

ASC is a rare subtype of cholangiocarcinoma with more aggressive clinical and pathological features than other cholangiocarcinomas, and the prognosis of patients with hepatic ASC is extremely poor. Kobayashi et al. [6] reported a mean survival time for patients with hepatic ASC of 8.7 months, and the overall 1-, 2-, and 3-year survival rates after surgery were $38.5,16.2$, and $10.8 \%$, respectively. Uenishi et al. [15] reported a 1-year survival rate for intrahepatic cholangiocarcinoma of $68 \%$, indicating that the prognosis of patients with primary hepatic ASC was poorer than that of patients with the common type of hepatic cholangiocarcinoma.

Chronic inflammation of the biliary tree is thought to be caused by PSC, congenital biliary abnormalities, and hepatolithiasis. The pathogenesis of ASC is still unclear, but it may be caused by continuous irritation of the bile ducts by chronic inflammation or associated with congenital cysts of the biliary tract [16]. Barr and Hancock [8] and Hamaya et al. [17] found no normal epithelium in the tumors they observed, which contained only AC, AC metaplasia, and SCC components, leading them to suggest that ASC may arise from squamous metaplasia of AC cells. In the present case, recurrent acute inflammation due to PSC and/or retrograde cholangitis due to placement of the biliary stent initially led to chronic inflammation, which may then have been responsible for metaplastic changes in the biliary epithelium. The AC component was, therefore, located near the intrahepatic bile duct and was surrounded by the SCC component, with a transitional area between the 2 components.

Liver tumors in patients with PSC include cholangiocarcinoma, hepatocellular carcinoma, metastatic liver tu-

Hepatic Adenosquamous Carcinoma with PSC mor, and liver abscess $[18,19]$. Imaging of the tumor as a low-density lesion surrounded by a highly enhanced rim on CT may resemble the findings for cholangiocarcino$\mathrm{ma}$, metastatic liver disease, and liver abscess. ASC is a rare subtype of cholangiocarcinoma, and an accurate prebiopsy diagnosis by radiological imaging was difficult in this case. However, echo-guided ultrasound biopsy was useful for diagnosis, and echo-enhanced tissue sampling enabled biopsy of the optimal part of the tumor, avoiding the necrotic area.

In conclusion, this is the first report describing resection of a hepatic ASC in a patient with PSC. Patients with PSC should be recognized as being at risk of hepatic ASC, in addition to general cholangiocarcinoma, hepatocellular carcinoma, and metastatic liver tumor.

\section{Statement of Ethics}

Written informed consent was obtained from the patient for publication of this case report.

\section{Disclosure Statement}

The authors declare that they have no competing interests.

\section{Funding Sources}

No funding was received.

\section{Author Contributions}

K.Y. drafted the manuscript. A.N., S.O., K.K., K.M., and T.M. worked on clinical data acquisition. H.I. contributed to the treatment of the patient. M.T., I.M., Y.T., and M.K. revised the manuscript critically for important intellectual content. T.C. performed the pathological analysis. All authors read and approved the final manuscript.

References

1 Fevery J, Henckaerts L, Van Oirbeek R, Vermeire S, Rutgeerts P, Nevens F, Van Steenbergen W: Malignancies and mortality in 200 patients with primary sclerosering cholangitis: a long-term single-centre study. Liver Int 2012; 32:214-222.

2 Bergquist A, Ekbom A, Olsson R, Kornfeldt D, Loof L, Danielsson A, Hultcrantz R, Lindgren S, Prytz H, Sandberg-Gertzen H, Almer S, Granath F, Broome U: Hepatic and extrahepatic malignancies in primary sclerosing cholangitis. J Hepatol 2002;36:321-327. 
3 Nakajima T, Kondo Y: A clinicopathologic study of intrahepatic cholangiocarcinoma containing a component of squamous cell carcinoma. Cancer 1990;65:1401-1404.

4 Nishioka T, Kubo S, Tanaka S, Wakasa K, Takemura S, Kinoshita M, Hamano G, Kuwae Y, Shibata T, Suehiro S: Outcomes of hepatic resection in intrahepatic cholangiocarcinoma patients with diabetes, hypertension, and dyslipidemia: significance of routine follow-up. Liver Cancer 2016;5:107-120.

5 Yoh T, Hatano E, Yamanaka K, Nishio T, Seo S, Taura K, Yasuchika K, Okajima H, Kaido T, Uemoto S: Is surgical resection justified for advanced intrahepatic cholangiocarcinoma? Liver Cancer 2016;5:280-289.

6 Kobayashi M, Okabayashi T, Okamoto K, Namikawa T, Araki K: A clinicopathologic study of primary adenosquamous carcinoma of the liver. J Clin Gastroenterol 2005;39:544548.

7 Yeh CN, Jan YY, Chen MF: Adenosquamous carcinoma of the liver: clinicopathologic features in 12 patients and review of the literature. Int Surg 2002;87:125-129.

8 Barr RJ, Hancock DE: Adenosquamous carcinoma of the liver. Gastroenterology 1975;69: $1326-1330$
9 Hayashi T, Mizuki A, Yamaguchi T, Hasegawa T, Kunihiro T, Tsukada N, Matsuoka K, Orikasa H, Yamazaki K: Primary adenosquamous carcinoma of the liver which produces granulocyte-colony-stimulating factor and parathyroid hormone related protein: association with leukocytosis and hypercalcemia. Intern Med 2001;40:631-634.

10 Takahashi H, Hayakawa H, Tanaka M, Okamura K, Kosaka A, Mizumoto R, Katsuta K, Yatani R: Primary adenosquamous carcinoma of liver resected by right trisegmentectomy: report of a case and review of the literature. J Gastroenterol 1997;32:843-847.

11 Yeh CN, Jan YY, Chen MF: Adenosquamous carcinoma of the liver: clinicopathologic study of 10 surgically treated cases. World J Surg 2003;27:168-172.

12 Yokota H, Matoba M, Tonami H, Hasegawa T, Saito H, Kurose N: Imaging findings in primary adenosquamous carcinoma of the liver: a case report. Clin Imaging 2007;31:279-282.

13 Bergquist A, Said K, Broome U: Changes over a 20-year period in the clinical presentation of primary sclerosing cholangitis in Sweden. Scand J Gastroenterol 2007;42:88-93.
14 Bonato G, Cristoferi L, Strazzabosco M, Fabris $\mathrm{L}$ : Malignancies in primary sclerosing cholangitis - a continuing threat. Dig Dis 2015;33(suppl 2):140-148.

15 Uenishi T, Hirohashi K, Kubo S, Yamamoto T, Yamazaki O, Kinoshita H: Clinicopathological factors predicting outcome after resection of mass-forming intrahepatic cholangiocarcinoma. Br J Surg 2001;88:969-974.

16 Ochiai T, Yamamoto J, Kosuge T, Shimada K, Takayama T, Yamasaki S, Ozaki H, Nakanishi Y, Mukai K: Adenosquamous carcinoma with different morphologic and histologic components arising from the intrahepatic bile duct: report of a case. Hepatogastroenterology 1996;43:663-666.

17 Hamaya K, Nose S, Mimura T, Sasaki K: Solid adenosquamous carcinoma of the liver. A case report and review of the literature. Acta Pathol Jpn 1991;41:834-840.

18 Kudo M: Surveillance, diagnosis, treatment, and outcome of liver cancer in Japan. Liver Cancer 2015;4:39-50.

19 Kudo M, Izumi N, Sakamoto M, Matsuyama Y, Ichida T, Nakashima O, Matsui O, Ku Y, Kokudo N, Makuuchi M: Survival analysis over 28 years of 173,378 patients with hepatocellular carcinoma in Japan. Liver Cancer 2016;5:190-197. 